NCU-HEP-k074

Aug 2018

\title{
Quantum Spacetime Pictures and Dynamics from a Relativity Perspective
}

\author{
Otto C.W. Kong \\ Department of Physics and Center for High Energy and High Field Physics, \\ National Central University, Chung-Li 32054, Taiwan \\ E-mail: otto@phy.ncu.edu.tw
}

\begin{abstract}
Based on an identified quantum relativity symmetry the contraction of which gives the Newtonian approximation of Galilean relativity, a quantum model of the physical space can be formulated with the Newtonian space seen in a way as the classical approximation. Matching picture for the observable algebra as the corresponding representation of the group $C *$-algebra, describes the full dynamical pictures equally successfully. Extension of the scheme to a Lorentz covariant setting and beyond will also be addressed. The formulation of quantum mechanics allows the theory to be seen in a new picture in line with the notion of a noncommutative spacetime.
\end{abstract}

* Talk presented at the 10th Jubilee International Conference of the Balkan Physical Union, Aug 26-30, 2018, Sofia, Bulgaria. 


\title{
Quantum Spacetime Pictures and Dynamics from a Relativity Perspective
}

\author{
Otto C.W. Kong ${ }^{1}$ \\ ${ }^{1}$ Department of Physics and Center for High Energy and High Field Physics, \\ National Central University, Chung-Li 32054, Taiwan \\ otto@phy.ncu.edu.tw
}

\begin{abstract}
Based on an identified quantum relativity symmetry the contraction of which gives the Newtonian approximation of Galilean relativity, a quantum model of the physical space can be formulated with the Newtonian space seen in a way as the classical approximation. Matching picture for the observable algebra as the corresponding representation of the group $C *$-algebra, describes the full dynamical pictures equally successfully. Extension of the scheme to a Lorentz covariant setting and beyond will also be addressed. The formulation of quantum mechanics allows the theory to be seen in a new picture in line with the notion of a noncommutative spacetime.
\end{abstract}

\section{INTRODUCTION}

In physics, experiments ultimately inform us as to what constitutes a good theoretical model of any physical concept: the physical space should be no exception. In a theory of particle dynamics like the Newtonian one, there is no physical picture of the physical space itself beyond that of the configuration space of a free particle (or the center of mass of a closed system of particles). The physical space is simply the totality of all possible positions for a physical object. Hence, the model for the physical space is not to be taken as independent of the physical theory. For a theory of particle dynamics, in particular, the model for the physical space assumed is only as good as the theory itself. We have seen how Einstein's theory of special relativity illustrate that the Newtonian model of the physical space is only to be taken as an inseparable part of the Minkowski spacetime. Quantum mechanics as it is to date, however, inherits with little critical revision many Newtonian conceptual notions. Most importantly, the Newtonian space model is still assumed, even though we are then forced to conclude that a physical object has no definite position. Our key question is if there is actually a different, better, model of the physical space for quantum mechanics. We want to see how the theory informs us as to what space is like, or what is the quantum model for it and how that is related to the more familiar Newtonian picture, which one must be able to retrieve as a limit or an approximation.

Quantum mechanics has position observables described by operators which are parts of the basic ingredients of an noncommutative algebra of observables. If the positions are modeled by a mathematical structure different from that of classical commutative observable algebra, the physical space they depict should be given by a different mathematical model. That naive thinking is behind efforts to construct quantum/noncommutative geometric models of the spacetime which has become quite a endeavor [1, 2, 3]. However, such efforts miss the true well established physical theory of the kind, namely simple quantum mechanics. Well, the position operators in quantum mechanics commutate among themselves. The latter seems to suggest that the quantum configuration space is like classical or commutative, only the quantum phase space is not. That perhaps is where the difficulty has been. In fact, we have a clear notion of the quantum phase space for a free particle as different from the classical one. It is given by the infinite dimensional (projective) Hilbert space. But there is no notion of the quantum configuration space. The main results, from our recent works [4, 5], we will sketch here is a full answer to all that. In a single statement, the project Hilbert space is the proper model for the physical space as behind quantum mechanics. As the space of pure states of the observable algebra, it is a mathematical structure dual to the latter [6] and hence can be seen as an alternative description of the noncommutative geometry. The configuration part and the momentum part of it are like the space and time parts of the Minkowski spacetime, they are not separable notions and can only truly be described independently in the 
Newtonian approximation. The Newtonian (configuration) space and phase space can be retrieved as approximations of the quantum model. As said, the model of the physical space is an integral part of the dynamical theory. Our analysis gives the complete relation between whole dynamics theories. Quantum mechanics is really a theory of particle dynamics on the quantum physical space, the classical approximation of which is Newtonian mechanics on the Newtonian space.

The configuration space and the phase space can be constructed as a representation space for the relativity symmetry. We can start from the Poincaré symmetry of special relativity with coset spaces $I S O(1,3) / S O(3) \times T(T$ denotes the time translation subgroup) and $I S O(1,3) / S O(1,3)$ giving the phase space for an Einstein particle and the Minkowski spacetime, respectively. The Newtonian approximation is to be retrieved as $c \rightarrow \infty$ which is best formulated as contraction limit [7, 8] of the relativity symmetry $I S O(1,3) \rightarrow G(3)$, the Galilean group [9]. For the case of quantum mechanics, one should take the $U(1)$ central extension of the Galilean group $\tilde{G}(3)$ as the true relativity symmetry. A contraction as like $\hbar \rightarrow 0$ limit trivializes the central extension and retrieves the classical results.

Our background framework is one of deformations of special relativity [10, 11, 12]. Symmetry deformation is essentially the inverse of symmetry contraction. The latter can be much more rigorously implemented to all aspects of the theory formulated from the symmetry. We have in mind an ultimate quantum relativity symmetry that is kind of fully noncommutative and stable against further deformation and having essentially all fundamental constants $G$, $\hbar$, and $c$ incorporated as like structural constants of the Lie algebra. The whole mathematical scheme as we illustrate for the full dynamics quantum to classical can be performed for the candidate theories at any level of the relativity symmetries as some contraction of the original. A specific contraction exactly gives a theory as an approximation to the one at the higher level. In fact, we believe to any dynamical theory of noncommutative/quantum spacetime geometry, our results may serve as the crucial first link from the bottom-up.

In the next section, we will give a sketch of the scheme of mathematical formulation which is expected to work in general. After that we will present a bit of details for the explicit case of quantum mechanics and its Newtonian approximation.

\section{SCHEME OF MATHEMATICAL FORMULATION}

A plausible picture of the full relativity symmetry contraction scheme can be given as

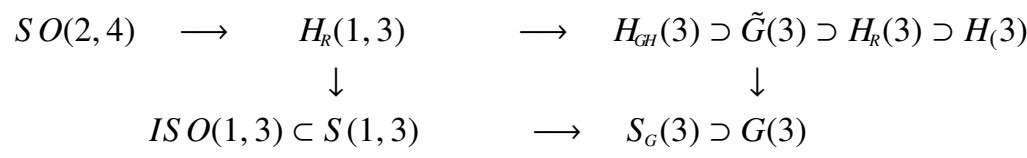

The $S O(2,4)$ symmetry has the Lorentz symmetry $S O(1,3)$ plus nine generators which can be seen as two fourvectors for essentially $X_{\mu}$ and $P_{\mu}$ and their commutator of the Heisenberg form. Commutators among the $X_{\mu}$ set, and also among the $P_{\mu}$ set, are $S O(1,3)$ generators. The limit the two four-vector sets each has mutually commutative components is the $H_{R}(1,3)$ symmetry, a Heisenberg-Weyl group with the $1+3$ sets of $X_{\mu}$ and $P_{\mu}$ supplemented by the rotation symmetry within each set. $S(1,3)$ is an extension of the Poincaré symmetry and is the classical limit of $H_{R}(1,3)$ with the Heisenberg commutators trivialized. Each arrow can be implemented as a contraction. The horizontal arrows apart from the first one is the $c \rightarrow \infty$ ones. Details of the contractions together with some discussion of the physics pictures as seen from the relevant coset spaces are presented in Ref.[13], illustrating a story consistent with all known physics. The $H_{R}(1,3)$ would give a Lorentz covariant quantum mechanics. Under the framework, the relativity symmetry for quantum mechanics has to be extended to the $H_{G H}(3)$ group and for Newtonian mechanics the $S_{G}(3)$ group. For the relevant representation of focus here, the extensions do not matter. We have only to focus on an irreducible representation of the $H(3)$ subgroup for the quantum cases and its classical limit. The rest of the group acts on the representation in an essentially trivial manner. For example, such a representation is the familiar spin zero time independent representation of the $\tilde{G}(3)$ group. Note that technically, we use $X_{i}$ in the place of the generators for Galilean boosts which should be taken as $m X_{i}$ for a particle of mass $m$.

With a Lie group serving as the relativity symmetry, the model for the physical space is to be given by a representation. An irreducible unitary representation is naturally served as a cyclic topological irreducible $*$-representation of the group $C^{*}$-algebra which serve as the algebra of observables. Guided by the case of quantum mechanics, the project Hilbert space for such a representation may is the space of pure states for the algebra, obtainable from the GNS construction [14]. From the group theoretical point of view, the unitary representation can be formulated as a coherent state representation with each coherent state corresponds to point(s) on a coset space which may not be a 
vector space in general. Such spaces of pure state are typically Kähler manifolds with a symplectic structure. One has a natural formulation of Hamiltonian dynamics on the phase space which corresponds exactly to Schrödinger dynamics on the Hilbert space [15]. There is a Heisenberg picture of the dynamics as automorphism flows on the observable algebra. To implement a contraction, the effect of the contraction should be trace through the specific representation. The limiting results for the model of the physical space as well as the full dynamical theory can be retrieved.

Let us have a few more words on how the scheme works for the classical approximation to a quantum theory. At the classical level, we have a commutative observable algebra, irreducible representations of which has to be one dimensional. The quantum observable algebra has to contract to a commutative limit. The Hilbert space then reduces to the individual one dimensional subspaces each corresponds exactly to one pure state. Superposition principle is lost as superposition of two classical pure states is only a mixed state. The Hilbert space picture is one of Koopmanvon Neumann [16]. The 'projective Hilbert space' as the space of pure states reduces to the classical coset space; the canonical coherent states are essentially the classical states and the only ones that survive the contraction as pure states. The Heisenberg dynamics on the observable algebra reduces to Poisson dynamics. The latter is a familiar fact which is behind the idea of deformation quantization. From our relativity symmetry perspective, hence, the deformation in deformation quantization is really the deformation of the representation of the group $C^{*}$-algebra as a consequence of the deformation of the relativity symmetry group and the corresponding representation.

\section{THE PROJECTIVE HILBERT SPACE MODELS}

We write quantum expressions with the $\hbar=2$ units here. This choice gives the Heisenberg commutation algebra the 'unusual' form of

$$
\left[X_{i}, P_{j}\right]=2 i \delta_{i j} I
$$

while setting the minimal uncertainty product to unity. The (canonical) coherent state representation is given by

$$
\left|p^{i}, x^{i}\right\rangle=U\left(p^{i}, x^{i}\right)|0\rangle \equiv e^{-i \theta} U\left(p^{i}, x^{i}, \theta\right)|0\rangle
$$

where

$$
U\left(p^{i}, x^{i}, \theta\right) \equiv e^{i x_{i} p^{i} \hat{l}} e^{i \theta \hat{I}} e^{-i x^{i} \hat{P}_{i}} e^{i p^{i} \hat{X}_{i}}=e^{i\left(p^{i} \hat{X}_{i}-x^{i} \hat{P}_{i}+\theta \hat{I}\right)},
$$

$|0\rangle \equiv|0,0\rangle$ is a fiducial normalized vector, $\hat{X}_{i}$ and $\hat{P}_{i}$ are representations of the generators $X_{i}$ and $P_{i}$ as self-adjoint operators on the abstract Hilbert space $\mathcal{H}$ spanned by the $\left|p^{i}, x^{i}\right\rangle$ vectors, and $\hat{I}$ is the identity operator representing the central generator $I$. $\left(p^{i}, x^{i}, \theta\right)$ corresponds to a generic element of the Heisenberg-Weyl $H(3)$ group as

$$
W\left(p^{i}, x^{i}, \theta\right)=\exp \left[i\left(p^{i} X_{i}-x^{i} P_{i}+\theta I\right)\right]
$$

with

$$
W\left(p^{\prime i}, x^{i}, \theta^{\prime}\right) W\left(p^{i}, x^{i}, \theta\right)=W\left(p^{\prime i}+p^{i}, x^{\prime i}+x^{i}, \theta^{\prime}+\theta-\left(x_{i}^{\prime} p^{i}-p_{i}^{\prime} x^{i}\right)\right) .
$$

Note that we have $p^{i}$ and $x^{i}$ here corresponding to half the expectation values of $\hat{P}_{i}$ and $\hat{X}_{i}$. On the wavefunctions $\phi(p, x) \equiv\langle p, x \mid \phi\rangle$, we have (suppressing the $i$-index)

$$
\begin{aligned}
& \langle p, x|\hat{X}| \phi\rangle=\hat{X}^{L} \phi(p, x), \\
& \langle p, x|\hat{P}| \phi\rangle=\hat{P}^{\downarrow} \phi(p, x),
\end{aligned}
$$

where

$$
\begin{aligned}
& \hat{X}^{L}=x+i \partial_{p}, \\
& \hat{P}^{L}=p-i \partial_{x},
\end{aligned}
$$

and

$$
U^{L}(p, x) \phi\left(p^{\prime}, x^{\prime}\right) \equiv\left\langle p^{\prime}, x^{\prime}|U(p, x)| \phi\right\rangle=\phi\left(p^{\prime}-p, x^{\prime}-x\right) e^{i\left(p x^{\prime}-x p^{\prime}\right)} .
$$


We can see again that $\hat{P}^{\iota}$ and $\hat{X}^{L}$ generate translations in $x$ and $p$, respectively. The wavefunction $\phi_{a}(p, x)$ for the coherent state $\left|p_{a}, x_{a}\right\rangle$ is given by

$$
\phi_{a}(p, x) \equiv\left\langle p, x \mid p_{a}, x_{a}\right\rangle=e^{i\left(p_{a} x-x_{a} p\right)} e^{-\frac{1}{2}\left[\left(p-p_{a}\right)^{2}+\left(x-x_{a}\right)^{2}\right]} ;
$$

in particular, the $|0,0\rangle$ state wavefunction is denoted by $\phi_{0}(p, x)$ and $\phi_{0}(p, x)=e^{-\frac{1}{2}\left(p^{i} p_{i}+x^{i} x_{i}\right)}$, which is a symmetric Gaussian of unit width. The expression $\left\langle p, x \mid p_{a}, x_{a}\right\rangle$ may also be taken as giving the overlap of two different coherent states. We denote the Hilbert space of wavefunctions by $\mathcal{K}$, on which we have $\frac{1}{\pi^{n}} \int d p d x \bar{\phi}(p, x) \phi^{\prime}(p, x)=\left\langle\phi|\hat{I}| \phi^{\prime}\right\rangle$ with $\hat{I}=\frac{1}{\pi^{n}} \int d p d x|p, x \backslash p, x|$, which keeps $\phi_{a}(p, x)$ as a normalized wavefunction $(n=3)$. Apart from a set of measure zero, all irreducible representations of $H(3)$ are essentially the same, up to rescaling of $\hat{P}^{t}$ and $\hat{X}^{t}$ which can be taken as a different choice of the physical unit to measure them.

The contraction to give the classical approximation is given by $X_{i}^{c}=\frac{1}{k} X_{i}$ and $P_{i}^{c}=\frac{1}{k} P_{i}$ with $k \rightarrow \infty$, hence $\left[X_{i}^{c}, P_{i}^{c}\right] \rightarrow 0$. With the state $\left|p_{i}, x_{i}\right\rangle$ re-labeled as $\left|p_{i}^{c}, x_{i}^{c}\right\rangle$ by the corresponding classical values, namely $p_{i}^{c}=\frac{1}{k} p_{i}$ and $x_{i}^{c}=\frac{1}{k} x_{i}$ taken as finite, we have

$$
\begin{aligned}
& \left\langle p_{i}^{\prime c}, x_{i}^{\prime c}\left|\hat{X}_{i}^{c}\right| p_{i}^{c}, x_{i}^{c}\right\rangle=\left[\left(x_{i}^{\prime c}+x_{i}^{c}\right)-i\left(p_{i}^{\prime c}-p_{i}^{c}\right)\right]\left\langle p_{i}^{\prime c}, x_{i}^{\prime c} \mid p_{i}^{c}, x_{i}^{c}\right\rangle \\
& \left\langle p_{i}^{\prime c}, x_{i}^{\prime c}\left|\hat{P}_{i}^{c}\right| p_{i}^{c}, x_{i}^{c}\right\rangle=\left[\left(p_{i}^{\prime c}+p_{i}^{c}\right)+i\left(x_{i}^{\prime c}-x_{i}^{c}\right)\right]\left\langle p_{i}^{\prime c}, x_{i}^{\prime c} \mid p_{i}^{c}, x_{i}^{c}\right\rangle
\end{aligned}
$$

with

$$
\left\langle p_{i}^{c}, x_{i}^{\prime c} \mid p_{i}^{c}, x_{i}^{c}\right\rangle=\exp \left[i k^{2}\left(x_{i}^{\prime c} p_{i}^{c}-p_{i}^{\prime c} x_{i}^{c}\right)\right] \exp \left[-\frac{k^{2}}{2}\left[\left(x^{c}-x^{c}\right)^{2}+\left(p^{\prime c}-p^{c}\right)^{2}\right]\right] \rightarrow 0
$$

and $\left\langle p_{i}^{c}, x_{i}^{c} \mid p_{i}^{c}, x_{i}^{c}\right\rangle=1$. Hence, the overlap between any two coherent states vanishes and the $\hat{X}_{i}^{c}$ and $\hat{P}_{i}^{c}$ are completely diagonal. Each $\left|p_{i}^{c}, x_{i}^{c}\right\rangle$ gives a one dimensional irreducible representation of the contracted symmetry. We will see that as a representation for the group $C^{*}$-algebra for the full relativity symmetry group, the observable algebra is given by functions of $\hat{X}_{i}$ and $\hat{P}_{i}$ and hence functions of $\hat{X}_{i}^{c}$ and $\hat{P}_{i}^{c}$ at the classical limit. In the language of $\mathcal{K}$, the coherent state wavefunction of Equation (9) goes to a delta function centered on $\left(p_{a}^{c}, x_{a}^{c}\right)$, with $\hat{X}^{c L}=x^{c}+\frac{i}{k^{2}} \partial_{p^{c}} \rightarrow x^{c}$ and $\hat{P}^{c L}=p^{c}-\frac{i}{k^{2}} \partial_{x^{c}} \rightarrow p^{c}$.

The $H(3)$ is isomorphic as a manifold to the coset space $\tilde{G}(3) / S O(3) \times T$. We illustrate also the contraction picture of the coset space as

$$
\left(\begin{array}{c}
d p_{c}^{i} \\
d x_{c}^{i} \\
d \theta \\
0
\end{array}\right)=\left(\begin{array}{cccc}
\omega_{j}^{i} & 0 & 0 & \bar{p}_{c}^{i} \\
0 & \omega_{j}^{i} & 0 & \bar{x}_{c}^{i} \\
-\frac{1}{k^{2}} \bar{x}_{c j} & \frac{1}{k^{2}} \bar{p}_{c j} & 0 & \bar{\theta} \\
0 & 0 & 0 & 0
\end{array}\right)\left(\begin{array}{c}
p_{c}^{j} \\
x_{c}^{j} \\
\theta \\
1
\end{array}\right) ;
$$

note that here $x_{c}$ and $p_{c}$ are group parameters satisfying $x_{c}=k x$ and $p_{c}=k p$ which are different from the coherent state labels $x^{c}$ and $p^{c}\left(p_{c} \hat{X}^{c}-x_{c} \hat{P}^{c}=p \hat{X}-x \hat{P}\right)$. At the classical limit of $k \rightarrow \infty$, the $\theta$ coordinate complete decouples and becomes irrelevant. The classical phase space coset of $\left(p_{c}, x_{c}\right)$ is isomorphic to the space of pure states $\left(p^{c}, x^{c}\right)$ for the observable algebra, and have the configuration space $x_{c}$ and momentum space $p_{c}$ as independent parts. One can start with the coset $\tilde{G}(3) / I S O(3) \times T$ with $I S O(3)$ being the subgroup generated by $J_{i j}$ and $X_{i}$. We have

$$
\left(\begin{array}{c}
d x^{i} \\
d \theta \\
0
\end{array}\right)=\left(\begin{array}{ccc}
\omega_{j}^{i} & 0 & \bar{x}^{i} \\
\bar{p}_{j} & 0 & \bar{\theta} \\
0 & 0 & 0
\end{array}\right)\left(\begin{array}{c}
x^{j} \\
\theta \\
1
\end{array}\right)
$$

the contraction limit of which also decouples $\theta$. The coset space can be taken to give 'coherent states' of $\left|x^{i}\right\rangle$ which are really position eigenstates. The Hilbert space is however unitary equivalent to $\mathcal{H}$ above. The Hilbert space picture, or that of the Schrödinger wavefunctions $\phi\left(x^{i}\right)$ can actually be used for which one can see that the momentum operator $-i \hbar \partial_{i}$ goes to the zero limit as $x$ is replaced by $x^{c}$ in the contraction. The projective Hilbert space of the reduced Hilbert space, or the space of pure states, then becomes again isomorphic to the classical coset of $x_{c}$. The picture cannot be used to look at the Newtonian dynamics which cannot be described on Newtonian space and functions on it alone. 


\section{DYNAMICS IN THE WWGM FORMALISM}

The most transparent mathematical formulation of the full dynamical theory from the relativity symmetry perspective is a Weyl-Wigner-Groenewold-Moyal (WWGM) formalism [17, 18, 19, 20, 21] formalism with wavefunctions on the coherent state basis as the starting point. In fact, the representation on $\mathcal{K}$ is really an irreducible component of the regular representation of $H(3)$ and the operators $\hat{X}_{i}^{L}$ and $\hat{P}_{i}^{L}$ the reduction of the left invariant vector fields. They can be written as $x_{i} \star$ and $p_{i} \star$, respectively, with the $\star$ of the Moyal star product given by

$$
\alpha \star \beta(p, x)=\alpha(p, x) e^{-i\left(\overleftarrow{(}_{p} \vec{\partial}_{x}-\overleftarrow{\partial}_{x} \vec{\partial}_{p}\right)} \beta(p, x) .
$$

$\alpha(p, x) \star=\alpha(p \star, x \star)$ as a differential operator is exactly the representation of elements of the group $C^{*}$-algebra on the Hilbert space $\mathcal{K}$. The formalism hence unifies the usual WWGM picture with the Hilbert space one. Observables are functions of the position and momentum operators $x_{i} \star$ and $p_{i} \star$ (Weyl ordering assumed), acting on the wavefunctions $\phi(p, x)$. An operator product $\alpha \star \beta \star$ can be taken as $(\alpha \star \beta) \star$ which is the notion of the Moyal star product. The Wigner distribution for a state is given by the projection operator $\rho_{\phi} \star$ and explicitly $\rho_{\phi}(p, x)(\star)=2^{2 n} \bar{\phi}(p, x) \star \phi(p, x)(\star)$. For a coherent state in particular, $\rho_{\phi_{a}}$ is a real Gaussian centered at the expectation values, as expected. Moreover, we have

$$
\operatorname{Tr}\left[\alpha \star \rho_{\phi}\right]=\frac{1}{2^{2} n} \frac{1}{\pi^{n}} \int d p d x \alpha(p, x) \rho_{\phi}(p, x)=\frac{1}{\pi^{n}} \int d p d x \bar{\phi}(p, x)[\alpha(p, x) \star \phi(p, x)] .
$$

We have worked through the WWGM formalism in details to obtain the explicit results [5] summarized above. However, one can really simply think about the whole story as writing down the full representation, again as a generic irreducible component of the regular representation of $H(3)$, for the group and the group $C^{*}$-algebra. Moreover, the regular representation can be seen as essentially the quasi-regular representation of the full relativity symmetry on the coset $(p, x, \theta)$ as given for the case of $\tilde{G}(3)$ above. It works as well for the extended group of $H_{G H}(3)$. That picture is the generic mathematical scheme that should work essentially for any Lie group with the right representation.

With the contraction, we have in terms of $p^{c}$ and $x^{c}$

$$
\star^{c} \sim \exp \left[\frac{-i \hbar}{k^{2}}\left(\overleftarrow{\partial}_{p^{c}} \vec{\partial}_{x^{c}}-\overleftarrow{\partial}_{x^{c}} \vec{\partial}_{p^{c}}\right)\right] \rightarrow 1
$$

i.e. the operator product reduces to $\alpha\left(p^{c}, x^{c}\right) \beta\left(p^{c}, x^{c}\right)$, namely the simple commutative product of functions as $\alpha(p, x) \star$ becomes $\alpha\left(p^{c}, x^{c}\right)$. The latter is the multiplicative operator on the Koopman-von Neumann Hilbert space formalism particularly useful for describing mixed states. A parallel picture at the quantum level can also be formulated with the notion of a Tomita representation [22, 23], which sees a density matrix (for a mixed state) as a vector in a Hilbert space (of operators). All that sits naturally in the functional algebra $C(p, x)$.

The general symmetry on $\mathcal{K}$ is described by the group of unitary transformations factored by its closed center of phase transformations. In particular, each one-parameter subgroup of the relativity symmetry transformations is realized as a (star-)unitary transformation in terms of real parameter $s$ as $U_{\star}(s) \star=e^{\frac{-i s}{2} G_{s} \star}$ with $G_{s} \star$ as the generator (the 2 is $\hbar) . G_{s}(p, x)$ is real as $G_{s} \star$ is Hermitian. For time translation, as a unitary transformation on $\mathcal{K}$, we have the Schrödinger equation of motion

$$
2 i \frac{d}{d t} \phi=G_{t} \star \phi
$$

The Heisenberg picture, for a generic $G_{s} \star$, gives

$$
\frac{d}{d s} \alpha \star=\frac{1}{2 i}\left[\alpha \star, G_{s} \star\right] .
$$

Dropping the last $\star$, the equation is equivalent to $\frac{d}{d s} \alpha=\frac{1}{2 i}\left\{\alpha, G_{s}\right\}_{\star}=\frac{1}{2 i}\left[\alpha \star G_{s}-G_{s} \star \alpha\right]$ where $\{\cdot, \cdot\}_{\star}$ is the Moyal bracket. $G_{t} \star$ is the Hamiltonian operator.

Under the contraction to the classical limit, the Schrödinger equation actually fails to give a proper limit. That is actually not surprising. Recall that the quantum Hilbert space for the pure states reduces to simple sum of one dimensional subspace of the coherent states which becomes essentially delta functions. Wavefunctions $\phi\left(p^{c}, x^{c}\right)$ other than the delta functions no longer describe pure states. In the picture of $\phi\left(p^{c}, x^{c}\right)$, the pure states are simply no 
continuous distributed. One can go to the Tomita representation which includes the mixed states and look at the Liouville equation as equation of motion for the density operator $\rho \star$, which for the special case of a pure state is equivalent to the Schrödinger equation. That has a proper limit as

$$
\frac{d}{d t} \rho\left(p^{c}, x^{c} ; t\right)=\frac{k^{2}}{2 i \hbar}\left\{G_{t}^{c}\left(p^{c}, x^{c}\right), \rho\left(p^{c}, x^{c} ; t\right)\right\}_{\star^{c}} \rightarrow\left\{G_{t}^{c}\left(p^{c}, x^{c}\right), \rho\left(p^{c}, x^{c} ; t\right)\right\}
$$

where $\{\cdot, \cdot\}$ is classical Poisson bracket $\{\alpha, \beta\}=\sum_{i}\left[\frac{\partial \alpha}{\partial x_{i}^{c}} \frac{\partial \beta}{\partial p_{i}^{c}}-\frac{\partial \alpha}{\partial p_{i}^{c}} \frac{\partial \beta}{\partial x_{i}^{c}}\right]$. Here, $G_{t}^{c}\left(p^{c}, x^{c}\right)$ is the classical Hamiltonian function. Similarly, the Heisenberg equation of motion has the right classical limit as

$$
\frac{d}{d t} \alpha\left(p^{c}, x^{c} ; t\right)=\frac{k^{2}}{2 i \hbar}\left\{\alpha\left(p^{c}, x^{c} ; t\right), G_{t}^{c}\left(p^{c}, x^{c}\right)\right\}_{\star^{c}} \rightarrow\left\{\alpha\left(p^{c}, x^{c} ; t\right), G_{t}^{c}\left(p^{c}, x^{c}\right)\right\} .
$$

Moreover, all that works for any generic Hamiltonian flow with generator $G_{s}$; Equation (18) gives the corresponding automorphism flow on the observable algebra the classical limit of which is given in the form of the Poisson bracket expression above.

We can define a

$$
\tilde{U}_{\star} \alpha \star=\mu(\alpha) \star=\bar{U}_{\star} \star \alpha \star U_{\star} \star,
$$

and see $\tilde{U}_{\star}$ as a (star-)unitary operator on some Hilbert space of operators. The Tomita representation corresponds exactly to such a picture, which gives on a density operator $\rho$ as a vector on the Hilbert space a 'Schrödinger equation' in the form

$$
\frac{d}{d s} \rho(s)=\frac{1}{2 i} \tilde{G}_{s} \rho(s)=\frac{1}{2 i}\left\{G_{s}, \rho(s)\right\}_{\star},
$$

which is the Liouville equation. Furthermore, we can actually look at $G_{s}$ and $\tilde{G}_{s}$ as independent symmetry generators and hence get a kind of doubled representation picture. For example, we have for the position and momentum operators

$$
\begin{array}{ll}
G_{-x^{i}} \star=p_{i} \star, & \tilde{p}_{i}=\tilde{G}_{-x^{i}}=2 i \partial_{x^{i}}, \\
G_{p^{i}} \star x_{i} \star, & \tilde{x}_{i}=\tilde{G}_{p^{i}}=2 i \partial_{p^{i}} .
\end{array}
$$

Similar fundamental set of operators was long ago introduced within the Koopman-von Neumann formulation [25]. The particularly interesting thing is the following classical limits

$$
\tilde{G}_{p^{c}}^{c}=i \hbar \partial_{p^{c}}, \quad \tilde{G}_{-x^{c}}^{c}=i \hbar \partial_{x^{c}},
$$

giving translations in $p^{c}$ and $x^{c}$. So, on the Koopman-von Neumann Hilbert space for classical mechanics, $G_{p^{c}}^{c}$ and $G_{-x^{c}}^{c}$ as well as all $G_{s^{c}}^{c}\left(s^{c}\right.$ may or may not equal to $\left.s\right)$ are diagonal while generators of the type $\tilde{G}_{s^{c}}^{c}$ are not. It is the latter class of generators that implement the classical relativity symmetries as like translations and rotations. For the Galilean (free particle) Hamiltonian, we have $\left(t^{c}=t\right)$

$$
\tilde{G}_{t}^{c}=\frac{-i \hbar}{m} p_{i}^{c} \partial_{x_{i}^{c}}
$$

which match exactly to the classical equation of motion as given in terms of the Poisson bracket.

\section{A NEW PICTURE FOR QUANTUM MECHANICS}

As a theory of particle dynamics on the quantum physical space, quantum mechanics can be seen in a different light which has a physical picture no less intuitive than classical mechanics. In a way, that fully resolves issue in the famous Einstein-Bohr debate which can be seen as paradoxical or counter-intuitive because they have been formulated on concepts of the position in space and other physical quantities described in terms of a mathematical model which only works well as the classical approximation. Using the right model, based first of all on the quantum model of 
the physical space and extended to all physical quantities as in the observable algebra, we have a successful abstract/mathematical formulation of physics at least consistent with common sense intuitive notion provided that the latter is strip of any familiar but otherwise abstract, hence not really physically intuitive, content from earlier versions of physical theory like the Newtonian mechanics.

The Newtonian space with its three dimensional Euclidean geometry, or for that matter any classical model of space(time) modeled by a finite dimensional commutative geometry is only part of a classical theory, which fails at the quantum level. Such commutative geometry is geometry modeled locally on products of the real number line. It has coordinate observables which can only be part of a commutative algebra. Taking the number of dimension to the infinite limit, the picture become nontrivial. However, there is really nothing intuitive about such finite dimensional nature of the physical space. That is to say, there is nothing intuitive in the statement that the position of a physical object, or the smallest indivisible part of one, can be specified with exact precision by three (or a finite number of) real numbers. The real number system is an algebraic system developed to model the notion of a continuum. It is not even the only possible model of the latter though. Physicists have been assuming all physical quantities are real-number valued. But that remains nothing more than an assumption, or a model that works well for classical physics. The real number answer is all practical measurements come from the real number scale/reading we ourselves put into the measuring apparatus in the calibration. And all such reading has uncertainties.

With quantum mechanics, we see that the observable algebra is noncommutative. It can be seen as a functional algebra $\alpha\left(\hat{P}_{i}, \hat{X}_{i}\right)$ of the three position and three momentum observables the first model of which are operators on the quantum Hilbert space. In the meantime, there has been much development in the mathematical theory of noncommutative geometry [24] essentially seeing the position and momentum operators as the noncommutative coordinates, here at least for the noncommutative phase space. The projective Hilbert space $C \mathcal{P}^{\infty}$ as the quantum phase space is the space of pure states dual to the observable algebra. Hence, it can be seen as an alternative description of the noncommutative phase space in the language of real number geometry with infinite dimension. In fact, it is easy to appreciate that the full physical content of any operator can be described, under a choice of coordinate system, by infinite number of real numbers. A naive example of the latter may be given by the set of matrix elements on a chosen orthonormal basis of the Hilbert space. We have now illustrated that the phase space is the right model for the physical space behind quantum mechanics. It is also interesting to note that the full observable algebra can also be formulated as an algebra of (so-called Kählerian) functions on $C \mathcal{P}^{\infty}$ [15]. What is missing is a more physical intuitive description of the geometry in terms of the noncommutative coordinates, on which we are now working.

The $C \mathcal{P}^{\infty}$ is a Kähler manifold hence a symplectic space. The Hilbert space itself is also a Kähler manifold. The Schrödinger equation is really a system of Hamiltonian equations for the in terms of coordinates on the Kähler manifold. In terms of the natural complex coordinates on the Hilbert space, given by $z^{n}=\left\langle z_{n} \mid \phi\right\rangle$ for an orthonormal basis $\left|z_{n}\right\rangle$, the real parts serves as configuration variables and the imaginary parts the conjugate momentum variables; the Hamiltonian function is $\frac{1}{2 \hbar}\langle\phi|\hat{H}| \phi\rangle$ taken as a function of $\left(p^{n}, q^{n}\right)$ with $z^{n}=q^{n}+i p^{n}$. Here, one can easily see that the phase rotation $|\phi\rangle \rightarrow e^{i \theta}|\phi\rangle$ as a basic (relativity) symmetry is a rotation on each of the $q^{n}-p^{n}$ plane, showing clearly the division between configuration or position type and momentum type of coordinates is only a choice of frame of reference. That is exactly like the nature of the space and time coordinates of the Minkowski spacetime in Einstein special relativity.

In the quantum/noncommutative physical space picture, a quantum particle has a definite position given by a point in the space, the solid mathematical description of which require an infinite number of real coordinates or six noncommutative coordinates. Such coordinates, in principle, can be determined with arbitrary finite precision. Such a particle also have fixed theoretical values for all observables without uncertainty. The value of each observable, however, has to be described by an element of a noncommutative algebra, or equivalently be an infinite set of real numbers. We should think about that as a kind of noncommutative number, which encodes a piece of quantum information about the particle. Uncertainties as in the Heisenberg uncertainty principle apply only to the best single real number description of the value of an observable on a state, that is the expectation value. In fact, in von Neumann, i.e. eigenvalue-answered, measurements, we need a good statistics from repeated measurements to get any good approximation to the expectation value. And with good enough statistics, we can obtain also the uncertainty as the standard derivation as well as the higher moments. The whole distribution, again a set of infinite real numbers, is what truly characterizes the value or the full information of the observable on the state. Better/optimal descriptions of all that is under study.

A word about the notion of Born probability is in order. The probability notion is quantum mechanics obviously is about the real number modeling of physical position observables and other quantities. Its implication on von Neumann measurements is only a statement about the statistical distribution. The latter physical result has also been illustrated 
as consequence of the decoherence induced by the measuring process. Adhering to the real number notion of all physical quantities and classical geometry for the physical space, the Born probability is the only workable way to interpret a quantum state in relation to the possible or allowable observable values. With the quantum physical space and noncommutative value of observables, there is no probability notion necessary. God does not play dices, only that he does not limit his mathematical tool used to build the physical universe to finite number of real numbers either.

\section{FURTHERING CONCLUDING REMARKS}

The notion of spacetime is the most fundamental of all physical concepts. Whatever fundamental theory we have, it would have some basic degrees of freedom which can be essentially thought of as a description of the spacetime, though such a spacetime model may be very different from the more familiar one. There would also be a notion of relativity symmetry as the background symmetry of the theory and the spacetime model, or the symmetry of reference frame transformations. We can thing of a special relativity for a kind of static background spacetime, and a general relativity for the fully spacetime. In this sense, a theory of quantum gravity would be more like a theory of geometrodynamics for a quantum spacetime, rather than some quantized dynamical theory on a classical spacetime.

One can certainly think of a quantum relativity symmetry beyond the usual group theory framework, such as using quantum groups. However, we see from our studies that Lie groups are amazingly powerful. A noncommutative Lie group has representations which extend to the group $C^{*}$-algebra are noncommutative. That looks like is enough to describe a noncommutative geometric space with any finite number of noncommutative coordinate observables. Formulating theory of the kind, with fully noncommutative $\hat{X}_{\mu}$ and $\hat{P}_{\mu}$ is already quite a challenge. However, it is also a very promising one from the relativity deformation/contraction perspective as sketched here. Quantum mechanics and its classical approximation has been formulated with the kind of relativity symmetry perspective very successfully, as we reported here. The mathematical scheme can be applied to the different Lie groups, hence solidly anchoring such theories to be developed on quantum mechanics and Newtonian mechanics.

The Planck constant characterizing the noncommutativity in quantum mechanics actually characterizes the constant holomorphic sectional curvature of the projective Hilbert space [15]. So, noncommutativity is curvature, which then suggests the fascinating idea of dynamical geometry implying dynamical noncommutativity.

Quantum field theory gives a very interesting spacetime perspective. Seen properly, all the quantum fields are more like degrees of freedom for the spacetime itself. Finding the quantum spacetime model incorporating that is another important challenge ahead.

Back to quantum mechanics, we have discussed how the theory can be seen in the light of noncommutative value for observables. Full practical implementation of the line of ideas may be like a Copernicus revolution to the physics of measurements. A measurement is just a controlled physical process to extract information about a physical system. The quantum world has information which is basically quantum. May be we can learn to extract and manipulate quantum information as kind of noncommutative numbers. There may be the days when physicists will calibrate our apparatus with noncommutative numbers.

\section{ACKNOWLEDGMENTS}

The author is partially supported by research grants number 107-2119-M-008-011 of the MOST of Taiwan.

\section{REFERENCES}

[1] P. Aschieri, C. Blohmann, M. Dimitrijević, F. Meyer, P. Schupp, and J. Wess, Classical and Quantum Gravity 22, 3511 (2005); P. Aschieri, M. Dimitrijević, F. Meyer, and J. Wess, Class. Quant. Grav. 23, 1883 (2006);

[2] P. Matlock, Phys. Rev. D 71, 126007 (2005); R. Banerjee, P. Mukherjee, and S. Samanta, Phys. Rev. D 75, 125020 (2007); P. Aschieri, F. Lizzi, and P. Vitale, Phys. Rev. D 77, 025037 (2008); M. Dimitrijević, V. Radovanović and H. Štefančić, Phys. Rev. D 86, 105041 (2012); C.A. Aguillón, A. Much, M. Rosenbaum and J.D. Vergara, J. Math. Phys. 58, 112301 (2017); M. Bojowald, S. Brahma, U. Buyukcam, and M. Ronco, arXiv: 1712.07413 [hep-th].

[3] H.-S. Yang, JHEP 05, 012 (2009); Int. J. Mod. Phys. A, 24, 4473-4517, (2009); Y.-G. Miao and S.-J. Zhang, Phys. Rev. D 82, 084017 (2010); Y.-G. Miao, Z. Xue, and S.-J. Zhang, Phys. Rev. D 83, 024023 (2011). 
[4] C.S. Chew, O.C.W. Kong, and J. Payne, Advances in High Energy Physics 2017, Special Issue on PlanckScale Deformations of Relativistic Symmetries, 4395918 (2017).

[5] C.S. Chew, O.C.W. Kong, and J. Payne, "Observables and Dynamics, Quantum to Classical, from a Relativity Symmetry Perspective”, arXiv:1703.04128 [quant-ph], NCU-HEP-k070 (2018).

[6] F.W. Shultz, Commun. Math. Phys. 82, (1982) 497-509 (1982); R. Cirelli, P. Lanzavecchia, and A. Manià, J. Phys. A: Math. Gen. 16, (1983) 3829-3835 (1983).

[7] D.N. Cho and O.C.W. Kong, Ann. Phys. 351, 275-289 (2014).

[8] R. Gilmore, Lie Groups, Lie Algebras, and Some of Their Applications (Dover, 2005); W.K. Tung, Group Theory in Physics (World Scientific, 1985).

[9] O.C.W. Kong and J. Payne, "Newtonian Limit from Symmetry Contraction of Special Relativity", NCUHEP-k067 (2018).

[10] O.C.W. Kong, Phys. Lett. B 665, 58-61 (2008).

[11] H.S. Snyder, Phys. Rev. 71, 38-41 (1947); C.N. Yang, Phys. Rev. 72, 874 (1974); R.V. Mendes, J. Phys. A 27, 8091-8104 (1994); C. Chryssomalakos and E. Okon, Int. J. Mod. Phys. D 13 1817-1850, (2004) ; ibid. 13, 2003-2034 (2004).

[12] G. Amelino-Camelia, Phys. Lett. B 510, 255-263 (2001); Int. J. of Mod. Phys. D 11, 35-59 (2002); J. Magueijo and L. Smolin, Phys. Rev. Lett. 88, 190403, (2002); Phys. Rev, D 67, 044017 (2003); J. Kowalski-Glikman and L. Smolin, Phys. Rev. D 70, 065020 (2004).

[13] O.C.W. Kong and J. Payne, "The First Physics Picture of Contractions from a Fundamental Quantum Relativity Symmetry Including All Known Relativity Symmetries, Classical and Quantum”, arXiv: 1802.02372 [hep-th], NCU-HEP-k071 (2018).

[14] F. Stroochi, An Introduction to the Mathematical Structure of Quantum Mechanics (World Scientific, 2008); F. David,Formalisms of Quantum Mechanics (Springer, 2015).

[15] R. Cirelli, A. Manià, and L. Pizzocchero, J. Math. Phys. 31, 2891-2897 (1990).

[16] B.O. Koopman, Proc. Natl. Acad. Sci. USA 17, 315-318 (1931); J. von Neumann, Ann. Math. 33, 587-642 (1932); ibid. 33, 789-791 (1932). See also H.-R. Jauslin and D. Sugny, "Dynamics of Mixed ClassicalQuantum Systems, Geometric Quantization, and Coherent States", in Mathematical Horizons for Quantum Physics, Edited by H. Araki et.al. (World Scientific, 2010).

[17] K.B. Wolf, "The Heiseberg-Weyl Ring in Quantum Mechanics", in Group Theory and Its Applications III, Edited by E.M. Loebl (Academic Press, 1975).

[18] F. Hansen, Rep. Math. Phys. 19, 361-381 (1984).

[19] J.M. Garcia-Bondía and J.C. Várilly, J. Math. Phys. 29, 869-879 (1988).

[20] D.A. Dubin, M.A. Hennings, and T.B. Smith, Mathematical Aspects of Weyl Quantization and Phase (World Scientific, 2000).

[21] C.K. Zachos, D.B. Fairlie, and T.L. Curtright (Eds.), Quantum Mechanics in Phase Space: An Overview with Selected Papers (World Scientific, 2005).

[22] M. Takesaki, Tomita's Theory of Modular Hilbert Algebras and Its Applications (Springer-Verlag, 1970).

[23] H.H. Grelland, Int. J. Theor. Phys. 32, 905-925 (1993).

[24] A. Connes, Noncommutative Geometry (Academic Press, 1994).

[25] A. Loinger, Ann. Phys. 20, 132-144 (1962). 